\title{
Las Biografías: un valioso recurso en las clases de Ciencias Naturales.
}

\section{Biographies: A valuable resource in Natural Science lessons}

Por: Rubinsten Hernández Barbosa ${ }^{1}$

Recibido: 08- 09-2010 Aceptado: 16-11-2010

\section{Resumen}

En el siguiente artículo se describe una experiencia de aula que resalta el valor didáctico que tiene el uso de las biografías de los hombres y mujeres que han aportado a la ciencia en los diferentes campos del conocimiento. En esta oportunidad, y teniendo como motivación el bicentenario del nacimiento de Charles Robert Darwin y los 150 años de la publicación de su obra cumbre, su vida y los planteamientos generales de su obra fueron objeto de estudio y reflexión por estudiantes del grado séptimo del Gimnasio Moderno de Bogotá, Colombia. Para cada una de las etapas que se desarrollaron se establecieron previamente las preguntas orientadoras, se acordaron las diferentes formas de trabajo y se determinaron las actividades, con el fin de mantener el interés por parte de los estudiantes y alcanzar los objetivos propuestos. Los resultados obtenidos en esta experiencia de aula permiten resaltar la importancia de las biografías como eje articulador de una propuesta de trabajo escolar y una forma distinta de abordar los contenidos de las ciencias naturales.

Palabras clave: Biografía, científicos, Darwin, evolución, historia de la ciencia, proyecto de aula.

\begin{abstract}
In the next article a classroom experience is described, which highlights the didactic value that the use of the biographies of the men and women who have contributed to science has in different fields of knowledge. In this opportunity and having as motivation the bicentenary of Charles Robert Darwin's birth and 150 years of the publication of his greatest work, his life and the general approaches of his work were object of study and reflection for students of the seven grade of the Gimnasio Moderno school in Bogotá, Colombia. For each of the stages that were developed previously, guiding questions were established, different ways of work were agreed and the activities were determined in order to maintain the student's interest and achieve the proposed objectives. The results obtained in this classroom experience make possible highlight the importance of biographies as the jointing axis of a school work proposal and a different way of tackling the contents of natural sciences
\end{abstract}

1 Magister en Didáctica de las ciencias, Universidad Autónoma de Colombia. Estudiante Doctorado en Educación, Universidad Distrital Francisco José de Caldas. E-mail: rhbjd@hotmail.com 
Keywords: Biography, scientists, Darwin, evolution, history of science, classroom project.

\section{INTRODUCCIÓN}

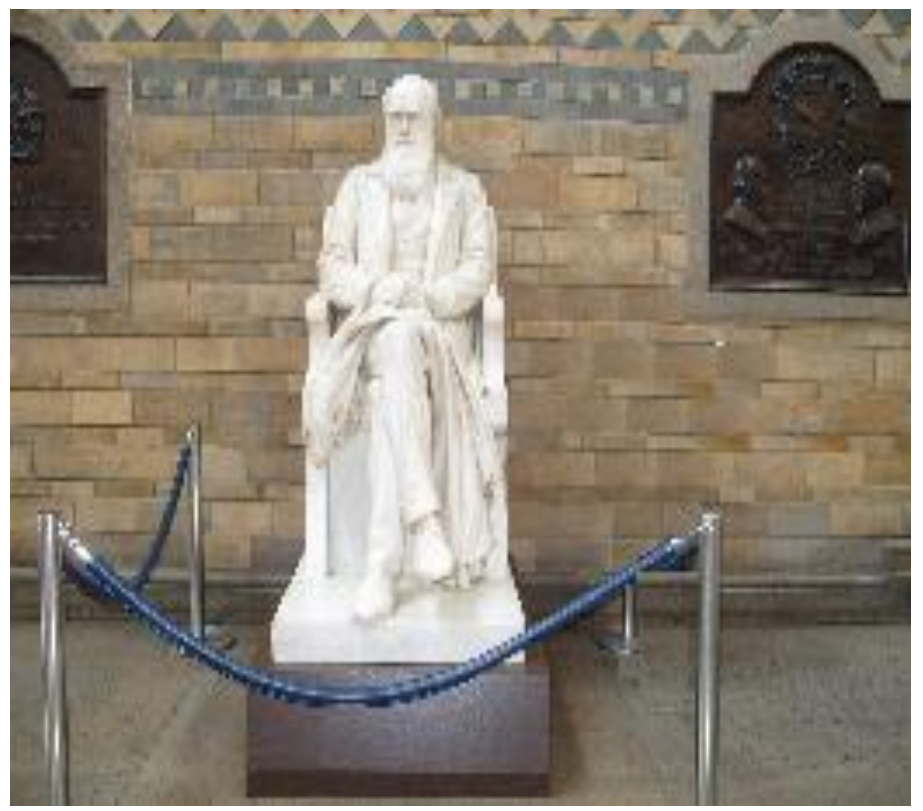

Estatua de Darwin en el Museo de Historia Natural de Londres².

En el 2009 las universidades, los institutos de investigación, especialmente aquellos que tiene como fundamento la biología, el gremio editorial de libros y revistas ${ }^{3}$ y los museos de Ciencias Naturales de muchas ciudades del mundo, entre otros, con motivo de la celebración del bicentenario del nacimiento de Charles Robert Darwin, y los 150 años de su obra fundamental: El Origen de las Especies por Medio de la Selección Natural, organizaron diferentes eventos y actividades que incluyeron exposiciones, congresos, presentaciones de libros, videos ${ }^{4}$ y conferencias sobre la vida y obra de quien propusiera la teoría evolutiva.

En el contexto escolar, los docentes de ciencias naturales no pudimos ser ajenos a esta conmemoración. Surgieron entonces las preguntas ¿Cómo hacer partícipes de esta celebración a los estudiantes? ¿Cuál estrategia metodológica implementar para que los

2 Foto tomada por el autor en el Museo de Historia Natural de Londres en Julio de 2008. Desde esta fecha ya se sentían todos los preparativos para el bicentenario del natalicio de Charles Robert Darwin y los 150 años del Origen de las Especies por Medio de la Selección Natural. Es uno de los personajes emblemáticos de Inglaterra, de ahí que su figura, desde el 2000, aparece en los billetes de diez libras. En el 2009 se lanzó una moneda.

3 Como ejemplo de ello, y particularmente de las ediciones especiales de las revistas científicas especializadas, se pueden revisar The American Biology Teacher y Ludus vitalis. Revistas de circulación general como la National Geografic e investigación y ciencia, o revistas institucionales universitarias de nuestro medio; todas ellas se unieron a la celebración del denominado año de Darwin. También un sin número de libros, de variadas procedencias y con diferentes aspectos relacionados Darwin, fueron editados.

4 Para mencionar los que hizo Richard Dawkins, etólogo y profesor de la universidad de Oxford, acerca de Darwin para el canal cuatro de la televisión inglesa. Se pueden encontrar en internet. 
estudiantes conozcan sobre la vida y la obra de Darwin? ¿Cuáles actividades son las más adecuadas? En el intento de dar respuestas a estas preguntas surgió el proyecto de aula ${ }^{5}$ que se llevó a cabo con estudiantes del grado séptimo de Bogotá, Colombia y que se describe en este documento. Se parte de unas ideas generales del marco conceptual que sustenta el proyecto, luego se describen las etapas del mismo, y finalmente se hacen algunas consideraciones sobre los resultados obtenidos y los aspectos a tener presente al momento de realizar una propuesta similar.

La relevancia que tiene una perspectiva histórica en los procesos de construcción de conocimiento científico escolar, donde los contextos sociales, políticos, culturales y económicos entre otros, son relevantes de análisis, ha sido establecida por muchas investigaciones (Matthews, 1994; Pedrinaci, 1996). Su importancia ha sido determinada no solamente para la educación básica escolar ${ }^{6}$, sino también para la educación universitaria, especialmente en la formación de futuros profesores de ciencias naturales, ya que como señaló Clark y Peterson, en 1986, citado por Mellado y Carracedo, (1993), las visiones que el docente tenga sobre los diferentes aspectos relacionados con la ciencia, permean, a través de su praxis, las construcciones que sobre la misma hace el estudiante.

En esa misma perspectiva, y teniendo en cuenta que el desarrollo de la ciencia no es lineal, ni continuo y muchos menos acumulativo, es necesario que se considere que el progreso del conocimiento científico se gesta y se desarrolla en el marco de una propuesta socio-histórica en el que establece relaciones muy estrechas con diferentes campos de la acción humana. Es a partir de esa reflexión que ha tomado fuerza, en los últimos años, el papel de la historia y la filosofía de la ciencia en la enseñanza de las ciencias, convirtiéndose en una línea de investigación de gran relevancia en la didáctica de las ciencias (Gil, 1993).

Se considera que la enseñanza de las ciencias en el contexto escolar, con una perspectiva histórica, ayuda a una comprensión de su naturaleza, de su génesis, de su impacto y de las repercusiones en la sociedad, de los obstáculos que se presentan en su proceso de desarrollo, lo cual implica entenderla como parte esencial de la cultura. El análisis de estos aspectos, y particularmente el relacionado con los obstáculos, favorece también procesos metacognitivos, pues ayuda a los estudiantes a reflexionar sobre sus propios procesos de pensamiento y a entender las dificultades que pueden tener al admitir otras concepciones diferentes a las que tienen en un momento determinado (Campanario, 1998).

Fernández (2000) ha señalado las ventajas que tiene la inclusión de la historia de las ciencia en los currículos escolares: fomenta actitudes positivas hacia la ciencia, muestra las relaciones entre Ciencia, Tecnología y Sociedad, pone de relieve la historicidad y la dimensión humana de la ciencia, ayuda a comprender la naturaleza, los métodos y evolución de la ciencia y, por su puesto, ayuda a comprender mejor los contenidos

5 La pedagogía de proyectos tiene su razón de ser en el desarrollo de proyectos de aula, los cuales pueden originarse por diferentes fuentes: por el azar, por sugerencia de los estudiantes, por iniciativa de las directivas de la institución o de los profesores, como en esta oportunidad. El tiempo también puede variar. En este caso se planeó para dos semanas, pero dado el interés de los estudiantes y de las actividades que se llevaron a cabo, el tiempo total fue de cuatro semanas.

${ }^{6}$ Para el caso colombiano, se entiende por educación básica, la educación primaria y el bachillerato. 
científicos. ¿Pero qué tipo de historia de la ciencia? Al respecto retomo las ideas de Castro, interpretando a Bachelard7; anotando que se necesita una historia de la ciencia crítica, que no se circunscriba a describir los hechos históricos, sino que éstos sean objeto de reflexión y juzgamiento. Que se comprenda que los procesos históricos implican continuidades y rupturas epistemológicas (Castro, 2009).

Es necesario entonces entender que la educación en ciencias naturales debe permitir, entre otras cosas, la reflexión sobre la representación del conocimiento científico, sus procesos experimentales, sus metodologías y sus aplicaciones en la resolución de problemas cotidianos de los individuos, los cuales se inscriben, se delimitan y condicionan en el marco de la cultura humana. Para ello es fundamental el análisis de los elementos que configuran el desarrollo de la ciencia: aspectos sociales, políticos, económicos, culturales y religiosos, entre otros (Ruiz, 2005).

Considerando lo planteado en los párrafos anteriores, y teniendo en cuenta que el docente de ciencias naturales cuenta con una amplia gama de estrategias metodológicas con el objetivo de facilitar el proceso de enseñanza-aprendizaje de las ciencias naturales, fue pertinente acercar a los estudiantes a los planteamientos de la teoría de la evolución a través de la biografía de su gestor. El uso de las biografías ${ }^{8}$ es una manera diferente, creativa y en ocasiones divertida, de aproximarse y abordar los contenidos de las ciencias naturales; ellas permiten resaltar los aspectos humanos, valorativos y formativos de quienes han aportado al desarrollo en los diferentes campos de la ciencia (Briceño, 2001).

El uso de las biografías como recurso metodológico tiene diferentes formas de llevarse a cabo dentro de un proyecto o en el desarrollo de una unidad didáctica. Al respecto, hay que anotar que la forma usada en esta experiencia de aula fue a través de las preguntas, las cuales fueron generadas tanto por los estudiantes como por el docente. Por eso esta propuesta también pretende resaltar la importancia que tiene la pregunta como generadora y orientadora de inquietudes, no sólo en los aspectos conceptuales, sino también en los metodológicos y actitudinales en el desarrollo de las ciencias. Además, las preguntas ayudan a guiar la búsqueda y selección de la información, aspecto que cada vez cobra mayor importancia en los procesos de investigación.

Dependiendo de los objetivos y de la manera como se formulan las preguntas, éstas pueden conllevar a desarrollar diferentes procesos cognitivos: memorización, descripción, interpretación, generalización y síntesis. En ésta y otras propuestas de trabajo, la pregunta tiene funciones importantes: hacer explícitos supuestos, creencias y conocimientos sobre un

\footnotetext{
7 Filosofo francés, quien considera el desarrollo del conocimiento en términos de obstáculos y rupturas epistemológicas. Anota que no puede haber conocimiento si no hay preguntas, éstas al ser resueltas, por diferentes metodologías, permiten la construcción de una teoría. Para Bachelard tener acceso a la ciencia es rejuvenecer espiritualmente, es aceptar una mutación brusca que ha de contradecir a un pasado. Para eso es necesario superar varios obstáculos.
}

8 Aquí se entiende por biografías todos aquellos textos que narran la vida de una persona dentro de un contexto histórico, resaltan los acontecimientos más importantes de ésta, señalan sus logros, aportes y fracasos entre otros, y destacan también las circunstancias sociales, políticas, económicas, culturales y familiares que le influyeron en el transcurso de su vida. 
tema, motivar la reflexión, mantener la motivación, explorar y abrir nuevas posibilidades de análisis y construcción de conocimiento. También se pueden clasificar en: contextualizadoras, contenedoras, valorativas, aclaratorias y profundizadoras (Villar, 2004).

La puesta en marcha de esta experiencia de aula tuvo tres objetivos:

1. Motivar a los estudiantes a conocer algunos aspectos sobre la vida de Charles Darwin.

2. Valorar la importancia de su trabajo en el campo de la biología9.

3. Implementar una propuesta metodológica usando las biografías como eje articulador de la misma.

A continuación se presenta cada una de las etapas del proyecto, se describen las actividades, se explicitan los objetivos, las preguntas orientadoras y se expone el valor didáctico ${ }^{10}$ que favoreció el uso de la biografía como recurso metodológico. Para cada una de las etapas se establecieron diferentes formas de trabajo y se desarrollaron distintas actividades, con el fin de mantener el interés por parte de los estudiantes y alcanzar los objetivos propuestos. Es importante anotar que esta propuesta de organización es sólo una guía, que los momentos que se describen pueden ser modificados y adaptados a los objetivos del docente, a la temática en la cual se inscribe el personaje seleccionado, a las necesidades y a los recursos de las instituciones escolares.

\section{METODOLOGÍA}

\section{a. Etapa de sensibilización}

Se inició con la lectura de un texto informativo ${ }^{11}$ que resaltaba la importancia de la celebración del bicentenario del natalicio de Darwin y los 150 años de la publicación $\boldsymbol{E l}$

9 Es importante anotar que los trabajos de Darwin abarcaron diferentes campos de la biología y de otras disciplinas. Sus intereses investigativos incluyeron diferentes organismos vivos: plantas como las orquídeas, animales como las palomas, los escarabajos, los insectos, los corales y los cirripedios (grupo de crustáceos), entre otros. A este último grupo de seres vivos le dedicó muchos años de estudio. Los resultados de sus investigaciones en este campo fueron publicadas en cuatro monografías: dos sobre grupos fósiles y dos sobre grupos vivientes, divididas a su vez en dos a los percebes y dos a los balanos. Lo anotado en la primera parte de la cita también se hace evidente en su extensa obra. Diario del viaje de un naturalista alrededor del mundo o El viaje del Beagle (1939), La estructura y distribución de los arrecifes de coral (1842), La estructura y distribución de los arrecifes de coral (1839), La fecundación de las orquídeas (1962), La variación de los animales y las plantas bajo domesticación El origen del hombre (1871), La expresión de las emociones en el hombre y en los animales (1872), Plantas insectívoras (1875), La formación del moho vegetal por la acción de los gusanos (1871). Su obra es más amplia. No todos sus textos han sido traducidos al idioma español y por lo tanto son menos conocidos en nuestro medio.

10 Se entiende por valor didáctico las posibilidades que ofrece las preguntas orientadoras pare el alcance de los objetivos por medio de actividades en las que se fortalecen las diferentes clases de contenidos: conceptuales, procedimentales y actitudinales.

11 El legado de Darwin. Texto publicado en Investigación y Ciencia. Enero 2009. Teniendo en cuenta el grado y las edades de los estudiantes, la escogencia de este texto obedeció a tres razones: la primera, aunque provenía de una revista especializada, el lenguaje y la forma de presentar el escrito sobre la vida y obra de Darwin era 
Origen de las Especies por Medio de la Selección Natural. Se aprovechó el título del documento para saber qué conocían sobre Darwin. El nombre de Darwin no les era indiferente ni desconocido, lo asociaron a la evolución, anotando que el hombre provenía del mono. Se procedió a leer el documento y dado que surgieron muchas inquietudes sobre los aspectos que se mencionaron en la lectura, se establecieron cuatro categorías a considerar en las siguientes etapas del proyecto: vida personal y familiar de Darwin, estudios realizados, viaje en el Beagle y la importancia de sus aportes a las ciencias naturales.

\begin{tabular}{|l|l|l|}
\hline \multicolumn{1}{|c|}{ Objetivo } & \multicolumn{1}{|c|}{ Valor didáctico } \\
\hline $\begin{array}{l}\text { Motivar a orientadoras } \\
\text { estudiantes a conocer } \\
\text { sobre la vida y obra del } \\
\text { padre de la teoría de la } \\
\text { evolución. }\end{array}$ & $\begin{array}{l}\text { ¿Quién fue Charles } \\
\text { Darwin? } \\
\text { ¿De dónde era? } \\
\text { ¿Por qué es importante } \\
\text { saber sobre Charles } \\
\text { Darwin? } \\
\text { ¿Cuáles fueron sus } \\
\text { planteamientos? }\end{array}$ & $\begin{array}{l}\text { Permite que los estudiantes } \\
\text { conozcan una parte del desarrollo de } \\
\text { la ciencia a través de la imagen del } \\
\text { personaje que se haya escogido, lo } \\
\text { cual depende de los objetivos del } \\
\text { curso, de las temáticas que se estén } \\
\text { abordando y de las circunstancias. } \\
\text { En este caso fue la celebración de los } \\
\text { 200 años del natalicio de Charles } \\
\text { Robert Darwin y los 150 años de su } \\
\text { obra fundamental: el origen de las } \\
\text { especies. }\end{array}$ \\
\end{tabular}

Tabla No 1. Objetivo y valor didáctico de las preguntas para la etapa de sensibilización.

\section{b. Vida de Charles Darwin}

Aprovechando el interés que suscitó el documento, se les invitó a conocer más sobre la vida de Darwin. Esta actividad se llevó a cabo en dos momentos; en el primero los estudiantes, de manera individual, buscaron y leyeron diferentes fuentes de información sobre Darwin. El segundo, teniendo en cuenta el plan de trabajo, los estudiantes fueron desarrollando cada uno de esos aspectos y organizándolos en el cuaderno, para luego socializarlo a los compañeros. En esta fase sobre la vida personal de Darwin, fue notorio el interés que los muchachos mostraron hacia los siguientes aspectos: el haber quedado huérfano muy pequeño (8 años), su gusto por coleccionar algunos animales, la influencia que tuvo su padre en los estudios ${ }^{12}$ que adelantó Darwin. Cada uno de esos aspectos fue objeto de

sencilla y amena. Aunque proporcionaba una información importante y permitía inferir otro tanto, había aspectos que no desarrollaba, y eso lo podían hacer los estudiantes; la segunda, las imágenes eran muy llamativas, y la tercera, presentaba una cronología de la vida antes y después de Darwin, lo cual, en su momento, se consideró importante para la consecución de uno de los objetivos del proyecto.

12 En 1825 inicia sus estudios de medicina en la Universidad de Edimburgo, Escocia. Allí se interesa principalmente por un curso de química y recibe clases de taxidermia. Un aspecto importante de esta etapa de su vida fue la relación que entabló con el zoólogo Robert Grant, con quien colecta ejemplares marinos. Insatisfecho por los resultados académicos y conocedor de su falta de vocación por la medicina, el padre de Darwin decide, otra vez, cuál sería el destino profesional de su hijo. Es así como en 1828 Charles Darwin comienza sus estudios eclesiásticos en el Christ' College de Cambridge. Allí conoce al reverendo John S. Heslow, con quien emprende 
análisis y reflexión; algunos de esas situaciones fueron puestos y analizados en la escena actual.

\begin{tabular}{|c|c|c|}
\hline Objetivo & $\begin{array}{c}\text { Preguntas } \\
\text { orientadoras }\end{array}$ & Valor didáctico \\
\hline $\begin{array}{l}\text { Conocer aspectos } \\
\text { generales de la vida } \\
\text { de Charles Robert } \\
\text { Darwin. }\end{array}$ & 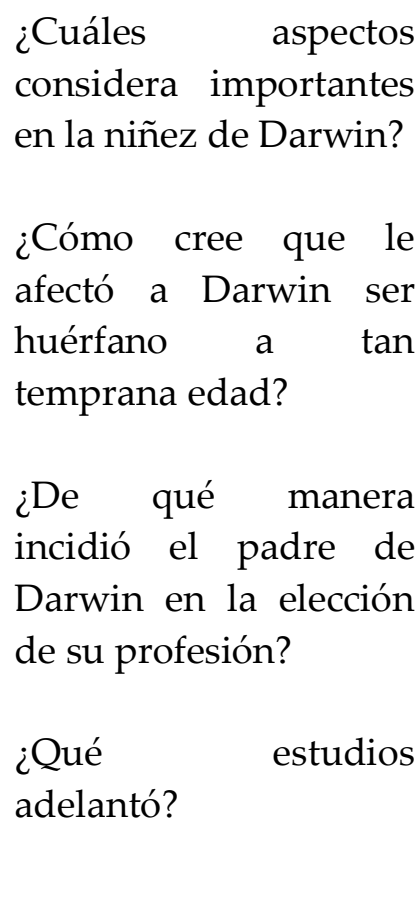 & $\begin{array}{l}\text { Destaca los aspectos humanos de los } \\
\text { científicos: la niñez, la familia, la } \\
\text { formación académica y las } \\
\text { circunstancias que rodean su vida } \\
\text { personal y profesional, las cuales, en } \\
\text { muchos casos, son determinantes en } \\
\text { la relación que establecen los } \\
\text { hombres y mujeres de la ciencia con } \\
\text { un campo de conocimiento. } \\
\text { Cuando se está reflexionado sobre } \\
\text { los aspectos personales de la vida } \\
\text { del científico (a) seleccionado, se } \\
\text { pueden introducir apartes de su } \\
\text { obra. }\end{array}$ \\
\hline
\end{tabular}

Tabla No 2. Objetivo y valor didáctico de las preguntas sobre la vida de Darwin.

\section{c. Personajes en la vida de Darwin}

En la lectura que se tomó como referente y en las informaciones que los estudiantes fueron seleccionando de la Internet y de otras fuentes de consulta, los nombres de Robert Darwin, Erasmus Darwin, John S. Henslow, Alexander Von Humboldt, Robert FitzRoy, Charles Lyell y Alfred Russel Wallace fueron apareciendo y tejiendo una serie de relaciones que los jóvenes, con ayuda del docente, fueron analizando con el objetivo de establecer los aportes y las incidencias de estas personas en la vida personal y profesional de Darwin. Se organizaron grupos y cada uno de ellos escogió un personaje, que previamente se había escrito en el tablero; luego se les invitó a responder las preguntas que aparecen en el cuadro, teniendo como material de apoyo las anotaciones del material de consulta y la lectura guía. Se abrió luego un espacio de socialización en la que un representante de cada grupo expuso el trabajo.

una amistad que duraría hasta su muerte. No se puede olvidar que Heslow, quien además de tener un buen concepto de Darwin como estudiante, y de ser su tutor, fue quien le sugiere tomar un curso de geología con Adam Sedgwick, el cual resultó ser de gran interés para Darwin. También fue Heslow quien lo relaciona con Robert FitzRoy, capitán del Beagle, 


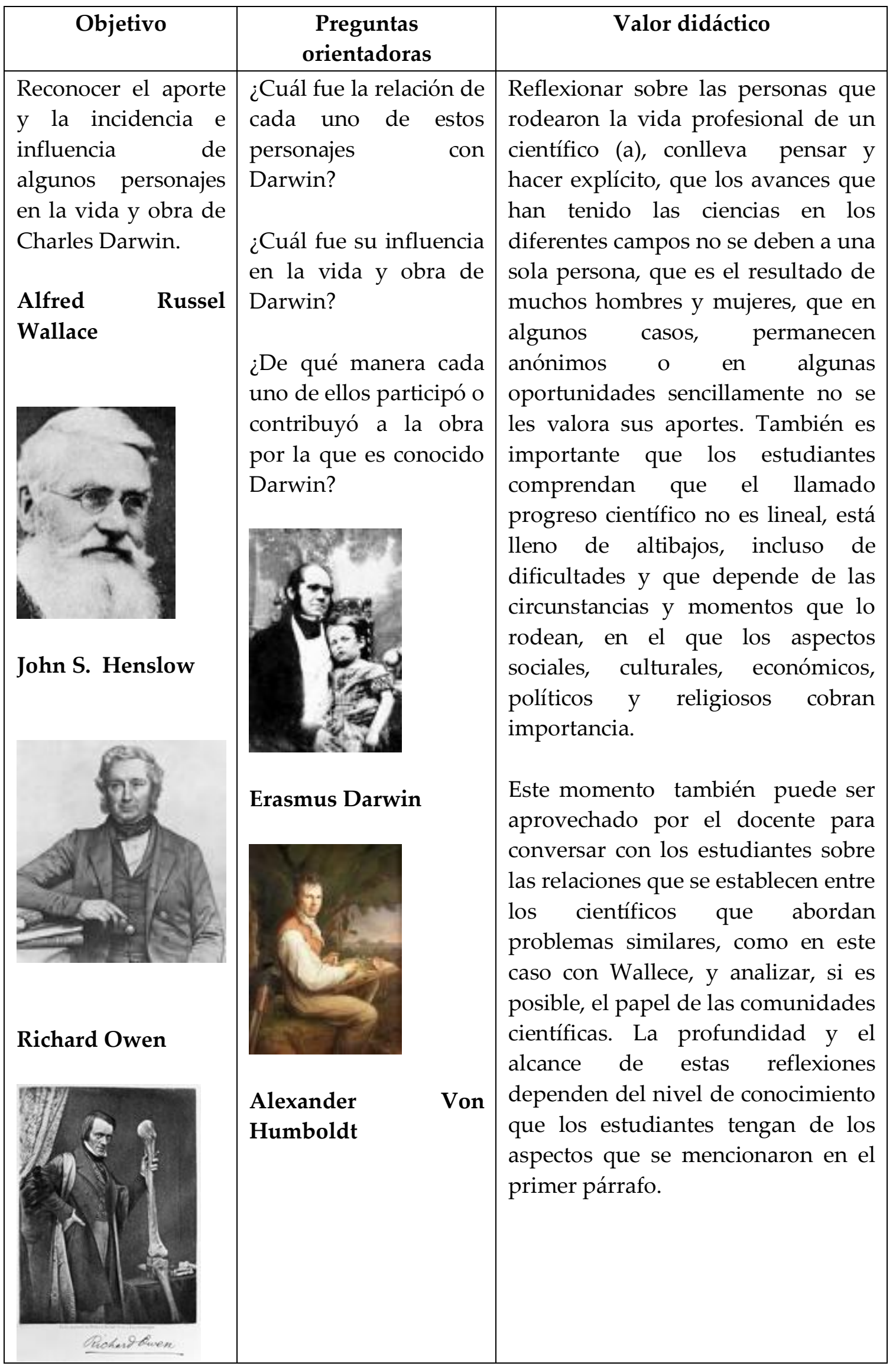

Tabla No 3. Objetivo y valor didáctico de las preguntas sobre la influencia de algunos personajes en la vida y obra de Charles Darwin. 


\section{e. Viaje en el Beagle}

Denominado por algunos el viaje que cambió la ciencia biológica ${ }^{13}$, al comando del capitán Robert Fitz Roy, el Beagle zarpó de Plymouth, Inglaterra, el 27 de diciembre de 1831 y arribó de regreso el 2 de octubre de 1836 (Strathern,1999). Fueron cerca de cinco años de aventuras, como lo denominaron los estudiantes. Usando un mapa del recorrido del Beagle se estableció la ruta general ${ }^{14}$; haciendo énfasis en los sitios visitados en Suramérica, luego en pequeños grupos, y habiendo seleccionado un lugar del recorrido, empezaron a revisar diferentes fuentes de consulta.

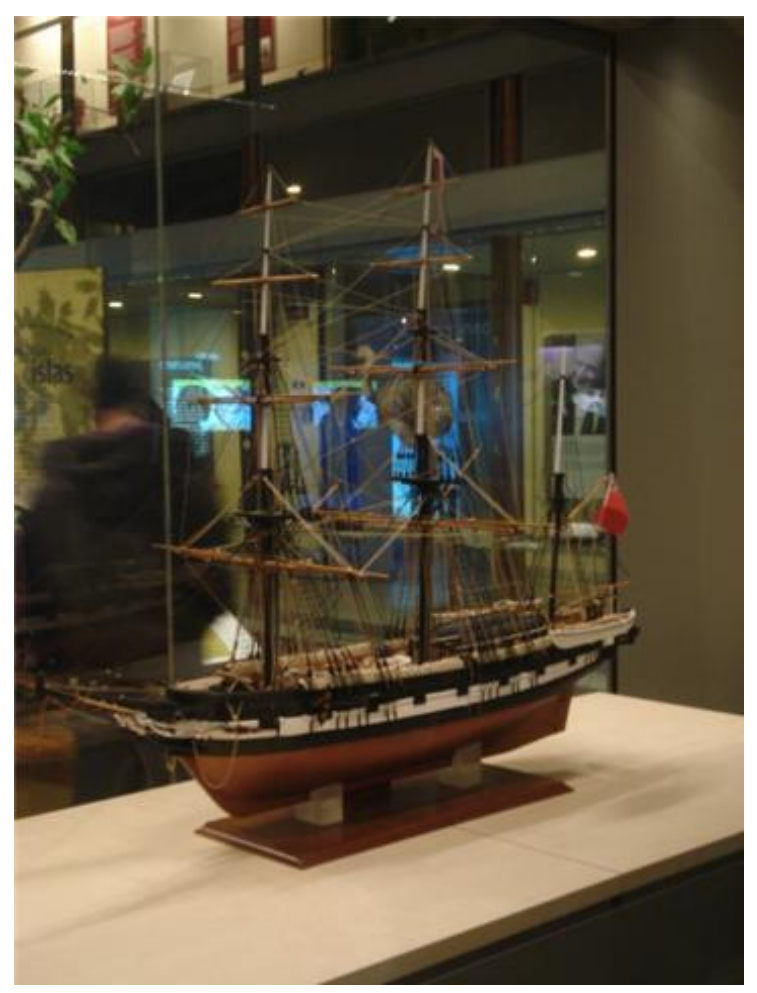

Réplica del Beagle en el Museo de Ciencias Naturales de Madrid, España ${ }^{15}$.

De acuerdo a lo sugerido por De santa et al (2005) el uso de las tecnologías de la informática y la comunicación son de gran ayuda, pues además de la información, a los estudiantes les

13 En todas las fuentes que el autor consultó sobre la vida de Darwin, es claro que el viaje del Beagle representó para Darwin un giro en su vida personal y profesional. A su regreso a Inglaterra es reconocido como un naturalista respetado y comienza su camino como científico. Sus planteamientos no estuvieron libres de críticas por un sector conservador de la sociedad y de la iglesia, especialmente. Incluso en algunos sitios sus libros fueron censurados y prohibidos. Tuvo que pasar mucho tiempo para que se le reconociera el alcance e importancia de sus planteamientos.

14 La ruta incluyó los siguientes lugares: Islas de Cabo Verde, Brasil, Argentina, Tierra de Fuego, las islas Malvinas, los Andes, las islas Galápagos, Tahití, la Bahía de las islas, Nueva Zelanda, Australia, Mauricio y Cabo de Buena Esperanza, entre otros.

15 Foto tomada en diciembre de 2009 por Freddy Robayo Salguero, compañero de viaje por la península ibérica. Por esa época el museo también hizo una exposición de fotos de los sitios visitados por Darwin en el Beagle. En la tienda del museo, la gran mayoría de los suvenires tenían la imagen de Darwin. 
suministra la consecución de material gráfico, el cual pueden adaptar y modificar de acuerdo a sus necesidades. También les facilita, como en este caso, programas para hacer presentaciones más dinámicas e interactivas. Cada grupo hizo su exposición sobre el lugar escogido, comenzando por Cabo Verde, África. De manera general, a los estudiantes les llamó la atención las diferentes peripecias que tuvo que vivir Darwin en cada uno de los lugares que visitó, lo enfermo que estuvo, los hallazgos en cada uno de los sitios y la colección de diferentes clases de especimenes, especialmente de fósiles, que envió a Inglaterra.

Una de las presentaciones que más les llamó la atención a la mayoría de los estudiantes, fue la que hizo el grupo que le correspondió Galápagos. Si bien les pareció interesante la información sobre este conglomerado de islas y vislumbraron la importancia que tuvo para Darwin en su propuesta sobre la teoría de la evolución, lo que más llamó su atención fueron las fotos que el grupo presentó sobre algunos animales endémicos, como los que se muestran en las siguientes imágenes.

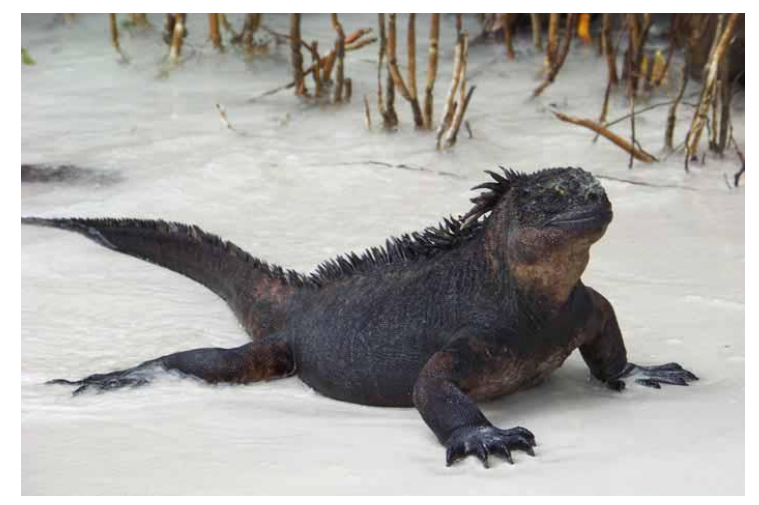

Iguana marina en Bahía Tortuga, Isla Santa Cruz ${ }^{16}$.

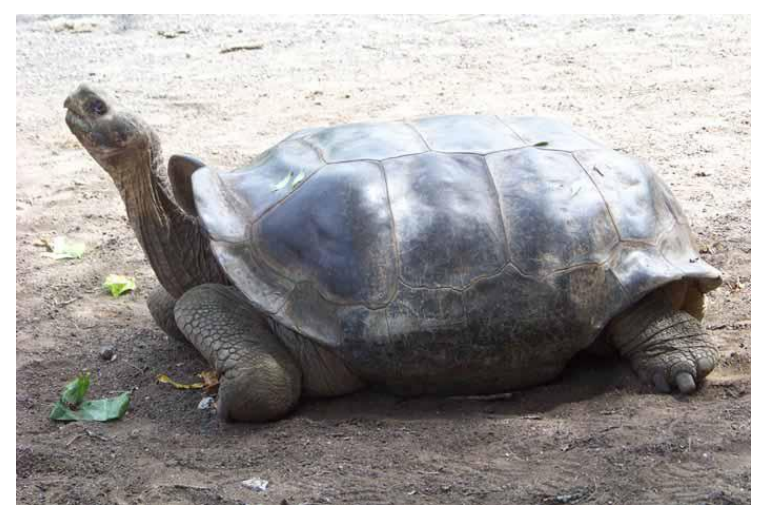

Tortuga galápago en el centro de crianza de la Isla Isabela ${ }^{17}$.

16 Foto tomada por Alejandro Castro en su viaje a las islas Galápagos. Publicada en su artículo Las islas Galápagos: Un viaje a través del espacio y el tiempo. Revista Biografía.

17 Foto tomada por Alejandro Castro en su viaje a las islas Galápagos. Publicada en su artículo Las islas Galápagos: Un viaje a través del espacio y el tiempo. Revista Biografía. 
En esta parte es fundamental hacer mención a El viaje del Beagle. Libro Publicado en 1839, su título original fue Diario y observaciones. De la mano del propio Darwin, este libro recoge las memorias de su viaje, las anotaciones y descripciones sobre los diferentes sitios visitados y sobre la gran cantidad de especímenes que recolectó durante su travesía.

Al finalizar las presentaciones y considerando el interés que los estudiantes expresaron por los aspectos relacionados con las aventuras que Darwin vivió durante su viaje en el Beagle, se les invitó a observar un video hecho por Unimedios ${ }^{18}$ de la Universidad Nacional, el cual les permitió complementar la información y tener una visión un poco más detallada e integral de lo que representó para Darwin cada uno de los lugares visitados.

\begin{tabular}{|c|c|c|}
\hline Objetivo & $\begin{array}{c}\text { Preguntas } \\
\text { orientadoras }\end{array}$ & Valor didáctico \\
\hline $\begin{array}{lr}\text { Describir } & \text { el } \\
\text { recorrido } & \text { del } \\
\text { Beagle, establecer } \\
\text { las características } \\
\text { de los lugares } \\
\text { visitados r y } \\
\text { determinar la } \\
\text { importancia de } \\
\text { los hallazgos en } \\
\text { cada uno de los } \\
\text { sitios. }\end{array}$ & $\begin{array}{l}\text { ¿Qué llevó a Darwin a } \\
\text { viajar en el Beagle? } \\
\text { ¿Cuál era la misión } \\
\text { principal del Beagle? } \\
\text { ¿Cuál sería el papel de } \\
\text { Darwin en esta } \\
\text { expedición? } \\
\text { ¿Qué lugares recorrió } \\
\text { el Beagle y qué } \\
\text { encontró en cada uno } \\
\text { de ellos? } \\
\text { ¿Cuánto tiempo duró } \\
\text { esta expedición? } \\
\text { ¿Qué aspectos de la } \\
\text { visita a Galápagos } \\
\text { llamó la atención de } \\
\text { Darwin y por qué? }\end{array}$ & $\begin{array}{l}\text { Destaca la importancia que tienen los } \\
\text { procesos de observación, descripción, } \\
\text { experimentación, comparación y } \\
\text { clasificación en un trabajo científico. } \\
\text { Además de lo anterior, y en este caso } \\
\text { particular del Viaje realizado por } \\
\text { Darwin, invita a repensar el papel que } \\
\text { juegan los dibujos, las descripciones, las } \\
\text { notas, las formas de preservar las } \\
\text { especies que Darwin recolectó, las } \\
\text { características biogeográficas de los } \\
\text { sitios visitados y ex plorados por la } \\
\text { expedición del Beagle }{ }^{19} \text {. }\end{array}$ \\
\hline
\end{tabular}

18 Este video se puede encontrar en: http://www.untelevision.unal.edu.co/multimedia/darwin/ . Además de los videos hechos por Dawkins, es importante mencionar los de History Chanel. También se pueden observar por internet.

19 Diario del viaje de un naturalista alrededor del mundo de Charles Darwin. Es un libro muy agradable e ininteresante. Darwin narra aspectos curiosos sobre las vivencias y los diferentes lugares que fueron visitados por él en el Beagle; además describe muchas especies de animales y de plantas hasta ese momento desconocidas en el mundo académico. 


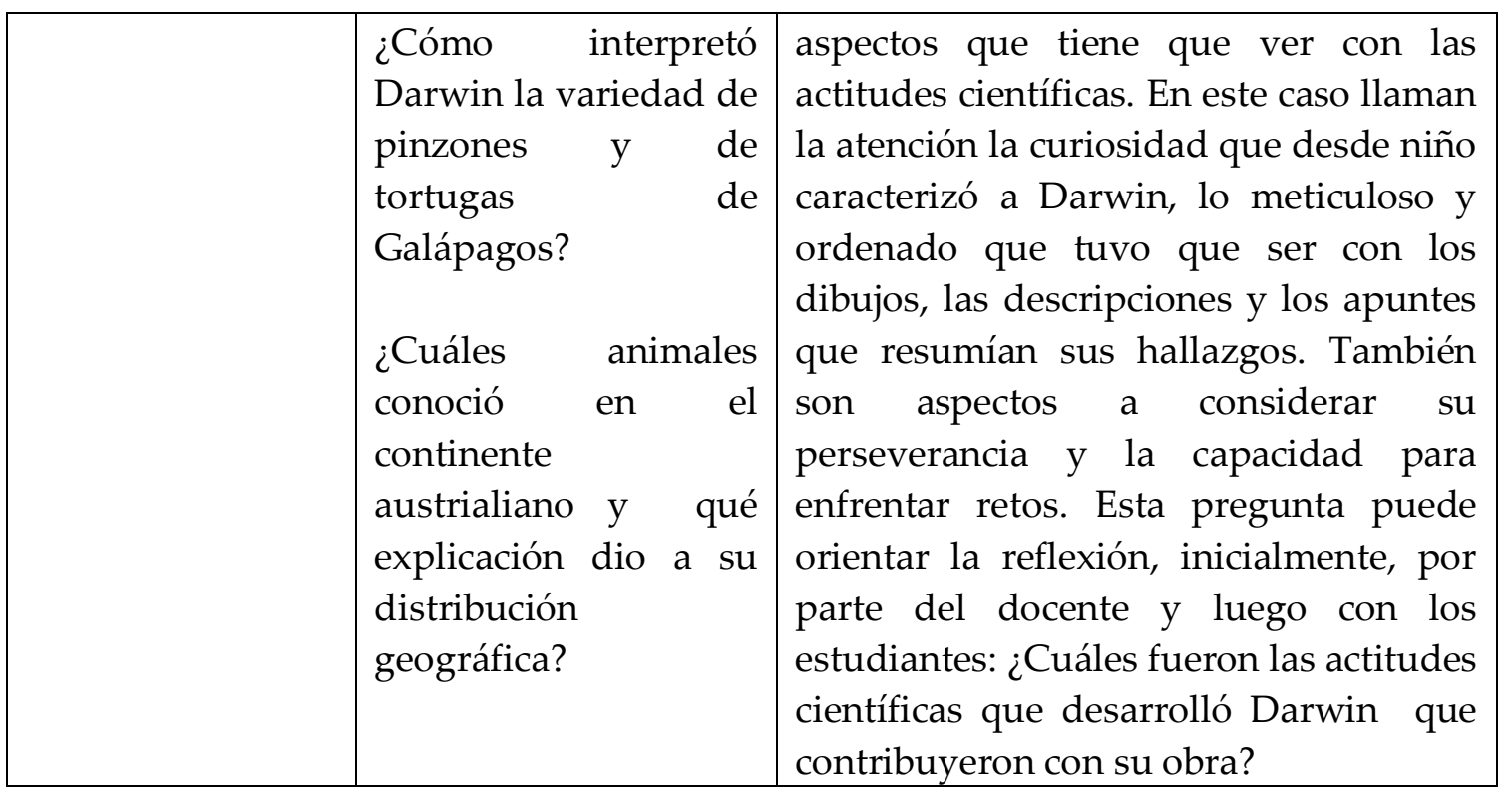

Tabla No 4. Objetivos y valor didáctico de las preguntas sobre el viaje que Charles Darwin hizo en el Beagle alrededor de Suramérica.

\section{f. Fósiles}

Esta etapa surgió después de las exposiciones sobre los lugares visitados por Darwin en el Beagle. El grupo que expuso sobre la visita a la Argentina hizo énfasis en el hallazgo de fósiles, cuya apariencia era muy similar a la de los actuales armadillos y osos perezosos. Los fósiles es un tema que llama mucho la atención de los jóvenes, situación que condujo a que se abriera un espacio solamente para conversar sobre ellos. Este momento fue muy interesante por dos razones: la primera, algunos estudiantes conocían sitios donde se han encontrado fósiles en Colombia (Ej: Desierto la Candelaria, Villa de Leyva) y otros los habían visto en los museos; la segunda, porque este espacio favoreció el compartir experiencias e intercambiar puntos de vista entre los alumnos sobre un tema de interés.

\begin{tabular}{|l|l|l|}
\hline \multicolumn{1}{|c|}{ Objetivo } & \multicolumn{1}{|c|}{$\begin{array}{c}\text { Preguntas } \\
\text { orientadoras }\end{array}$} & \multicolumn{2}{|c|}{ Valor didáctico } \\
\hline $\begin{array}{l}\text { Determinar la } \\
\text { importancia que } \\
\text { tuvo para Darwin el } \\
\text { hallazgo de fósiles }{ }^{20} .\end{array}$ & $\begin{array}{l}\text { ¿Cómo so fó forman los fósiles? } \\
\text { fósiles? }\end{array}$ & $\begin{array}{l}\text { Este tipo de actividades permite que } \\
\text { los estudiantes valoren la } \\
\text { importancia de las hipótesis y los } \\
\text { diferentes } \\
\text { contrastación. También puede } \\
\text { ayudar a reconocer diferentes }\end{array}$ \\
\hline
\end{tabular}

20 Culminada su travesía en el Beagle y de su exitoso regreso a Inglaterra, Darwin conoce a Richard Owen, un biólogo y especialista en anatomía comparada, quien, de manera voluntaria, se ofrece a estudiar los fósiles de grandes tamaños que él coleccionó en diferentes lugares en Suramérica y que fueron repartidos en varios museos de Londres y de Cambridge. El estudio de Owen determinó que los enormes fósiles eran roedores y perezosos, que estaban relacionados con especies que actualmente habitaban las zonas dónde fueron hallados. Se considera que este acontecimiento fue determinante en los fundamentos de la teoría de selección natural. 


\begin{tabular}{|l|l|l|}
\hline $\begin{array}{l}\text { factible de hallar } \\
\text { fósiles? }\end{array}$ & $\begin{array}{l}\text { metodologías en el trabajo } \\
\text { científico, en las que se resalta la } \\
\text { ¿Dónde encontró } \\
\text { fósiles Darwin? } \\
\text { formular y solucionar problemas. }\end{array}$ \\
¿Por qué se considera \\
importante el hallazgo \\
de fósiles en el \\
planteamiento Darwin \\
sobre la teoría de la \\
evolución? porán, de \\
¿En qué se parecen los \\
fósiles hallados por \\
Darwin y algunas \\
especies vivas de la \\
costa pacífica \\
Suramericana?
\end{tabular}

Tabla No 5. Objetivo y valor didáctico de las preguntas sobre los fósiles.

\section{d. Contexto sociocultural}

Este aspecto invitó a reflexionar sobre algunos aspectos que rodearon el momento histórico de la vida y obra del padre de la teoría evolutiva. Los estudiantes establecieron puntos de conexión y relación con temáticas que habían estudiado en el área de Ciencias Sociales. Fue así como se hizo una descripción de los aspectos socioculturales de la Inglaterra del siglo XIX, destacándose entre otras cosas, la posición privilegiada en lo político y económico que tenía este país en esos momentos y la importancia de las expediciones como una forma de mantener su hegemonía en otras partes del mundo y la consecución de nuevos recursos económicos. En el aspecto religioso, se hizo mención al papel de la iglesia y a su relación con la ciencia; para los estudiantes fue claro el control que esta ejercía en esos asuntos, ya que se consideraba la existencia de un ser superior que establecía un orden natural, en el que el ser humano tenía un papel fundamental.

\begin{tabular}{|c|c|c|}
\hline Objetivo & $\begin{array}{c}\text { Preguntas } \\
\text { orientadoras }\end{array}$ & Valor didáctico \\
\hline $\begin{array}{l}\text { Esbozar las } \\
\text { condiciones } \\
\text { socioculturale } \\
\text { s de la época } \\
\text { que vivió } \\
\text { Darwin. }\end{array}$ & 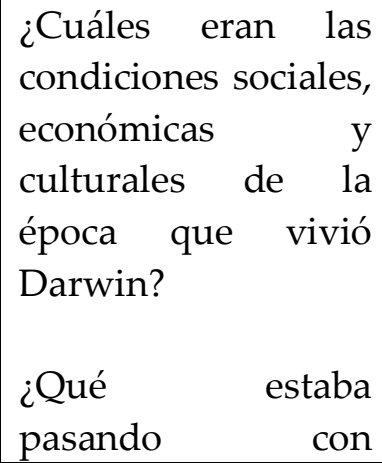 & $\begin{array}{l}\text { Analizar los aspectos políticos, económicos, } \\
\text { sociales, religiosos y culturales que } \\
\text { configuran un momento determinado de una } \\
\text { sociedad, especialmente porque en él se } \\
\text { suscitaron cambios importantes en el } \\
\text { desarrollo de un campo de conocimiento en } \\
\text { particular, conlleva a entender que la ciencia } \\
\text { es parte de la cultura y de la historia de la } \\
\text { humanidad. }\end{array}$ \\
\hline
\end{tabular}




\begin{tabular}{|l|l|l|}
\hline $\begin{array}{l}\text { Inglaterra en esos } \\
\text { momentos? }\end{array}$ & $\begin{array}{l}\text { Además, y dependiendo de la manera como } \\
\text { ¿Qué relación tenía } \\
\text { la iglesia con las } \\
\text { comunidades } \\
\text { científicas? }\end{array}$ & $\begin{array}{l}\text { aeden empezar a identificar las relaciones } \\
\text { entre la ciencia y sus diferentes campos de } \\
\text { acción, la sociedad, el ambiente y la } \\
\text { tecnología. También puede favorecer el } \\
\text { desmitificar una ciencia neutral y objetiva. }\end{array}$ \\
$\begin{array}{ll}\text { iCómo reaccionó la } \\
\text { iglesia frente a los } \\
\text { planteamientos de } \\
\text { Darwin? }\end{array}$ & $\begin{array}{l}\text { Aunque en esta experiencia particular este } \\
\text { aspecto no fue abordado, sería interesante } \\
\text { considerar, cuando se están abordando los } \\
\text { aspectos socioculturales, analizar el papel que } \\
\text { juegan los gobiernos, la procedencia de los } \\
\text { recursos económicos y las génesis de los } \\
\text { temas o campos de investigación. Al igual } \\
\text { que con otros aspectos que se mencionan en } \\
\text { las diferentes etapas, la profundidad de las } \\
\text { reflexiones dependen de las características de } \\
\text { los grupos con quienes se implemente esta } \\
\text { estrategia. }\end{array}$ \\
&
\end{tabular}

Tabla No 6. Objetivo y valor didáctico de las preguntas sobre las condiciones socioculturales de la época que vivió Darwin.

g. Aspectos conceptuales. Planteamientos de la teoría sobre la evolución y su importancia en el campo de la biología.

Esta fase no está separada de los otros momentos de la propuesta, por el contrario, se articula en un todo, ya que en este caso la biografía de Darwin fue usada como motivación para conocer aspectos relevantes de su vida personal y sobre sus planteamientos en la teoría de la evolución. También favoreció hacer un análisis de sus aportes, de la incidencia e impacto en la sociedad y reflexionar sobre la manera como cambió la perspectiva y visión de la biología.

\begin{tabular}{|c|c|c|}
\hline Objetivo & $\begin{array}{c}\text { Preguntas } \\
\text { orientadoras }\end{array}$ & Valor didáctico \\
\hline 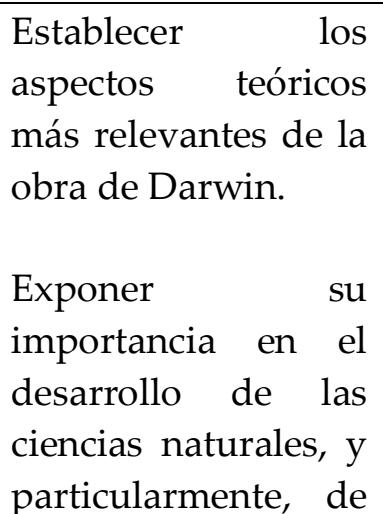 & $\begin{array}{l}\text { ¿Cuáles fueron los } \\
\text { planteamientos de } \\
\text { Darwin? } \\
\text { ¿En qué hechos se basó } \\
\text { Darwin para hacer sus } \\
\text { planteamientos? } \\
\text { ¿Qué estrategias usó } \\
\text { Darwin para poder } \\
\text { hacer sus análisis y } \\
\text { posteriormente }\end{array}$ & $\begin{array}{l}\text { El desarrollo de una teoría no es } \\
\text { lineal en el tiempo ni en sus avances. } \\
\text { A lo largo de este proceso se } \\
\text { presentan una serie de obstáculos, } \\
\text { que en el caso particular de la teoría } \\
\text { de la evolución, fue necesario } \\
\text { resistir para que fuera aceptada por } \\
\text { la comunidad científica: la forma de } \\
\text { concebir los procesos biológicos en } \\
\text { la época de Darwin, el papel }\end{array}$ \\
\hline
\end{tabular}




\begin{tabular}{|c|c|c|}
\hline la biología. & $\begin{array}{l}\text { proponer su teoría? } \\
\text { ¿Cómo se pueden } \\
\text { interpretar esos } \\
\text { planteamientos? } \\
\text { ¿Qué es evolución? } \\
\text { ¿Quiénes evolucionan? } \\
\text { ¿En qué se basa la } \\
\text { teoría de la evolución? }\end{array}$ & $\begin{array}{l}\text { decisivo de la iglesia y el impacto de } \\
\text { los planteamientos en un momento } \\
\text { donde el antropocentrismo era } \\
\text { decisivo en la forma de concebir el } \\
\text { mundo natural, son aspectos } \\
\text { centrales en la reflexión del trabajo } \\
\text { propuesto por Darwin. } \\
\text { Otro aspecto que se considera } \\
\text { necesario que el docente aproveche } \\
\text { para reflexionar con los estudiantes } \\
\text { es el entretejido que se hace entre los } \\
\text { diferentes campos disciplinares de la } \\
\text { ciencia. En este caso para los } \\
\text { estudiantes fue claro el aporte que } \\
\text { desde diferentes campos se hicieron } \\
\text { para que Darwin propusiera su } \\
\text { teoría: aspectos biogeográficos y } \\
\text { paleontológicos. } \\
\text { Además, abre un espacio para que } \\
\text { los estudiantes hagan conciencia del } \\
\text { esfuerzo que hombres y mujeres han } \\
\text { realizado en el desarrollo del } \\
\text { conocimiento científico y del } \\
\text { impacto que este ha tenido para la } \\
\text { sociedad desde diferentes } \\
\text { perspectivas, lo cual ha generado } \\
\text { cambios en la forma de pensar, } \\
\text { sentiry actuar del ser humano. }\end{array}$ \\
\hline
\end{tabular}

Tabla No 7. Objetivo y valor didáctico de las preguntas sobre las condiciones socioculturales de la época que vivió Darwin.

\section{h. Evaluación}

Un aspecto fundamental de todo proyecto es la evaluación. Aunque por organización del texto esté ubicada al final de las etapas de desarrollo, esta fue continua y se hizo desde diferentes miradas, teniendo en cuenta varios aspectos en cada una de ellas (búsqueda de información, cumplimiento de las tareas asignadas, la participación en los espacios de reflexión y la exposición), pero siempre prestando especial interés a las actitudes de los estudiantes, en la manera como estos desarrollaron cada una de las fases del proyecto y a las relaciones que fueron estableciendo y tejiendo sobre la vida y legado de Darwin.

El interés de los estudiantes fue creciendo, esto fue evidente en la forma entusiasta como se prepararon para las exposiciones, donde todos los grupos se mostraron interesados en consultar información sobre los sitios visitados por Darwin en el Beagle. Para la evaluación de este momento se tuvieron en cuenta los siguientes aspectos: la preparación y el desarrollo de la presentación (gráficos, tablas, dibujos), el manejo o forma de expresar las ideas sobre el 
tema, el trabajo en grupo y la apropiación de tareas en el mismo. Como trabajo final, y teniendo en cuenta todos los aspectos que fueron abordados sobre la vida y obra de Robert Charles Darwin, los estudiantes hicieron una línea del tiempo con estos datos. Se hizo en tres momentos: 1 . selección de los momentos más relevantes, 2. búsqueda y ampliación de la información, y 3. elección de fotos para ilustrar cada uno de los momentos escogidos.

Otro espacio muy interesante, dentro del proceso evaluativo, fue la comparación que los estudiantes hicieron entre lo que sabían de Darwin al comienzo y lo que ellos conocían después de haber realizado el proyecto. Este momento fue especial porque fueron los mismos estudiantes quienes, de manera abierta y sincera, manifestaron el cambio que habían tenido, con respecto a la información que conocían de Darwin y la que tienen ahora.

Además de las actividades propias del espacio de aula, los estudiantes de grado séptimo del Gimnasio Moderno, con quienes se llevó a cabo esta propuesta de trabajo, en el marco de la Semana de las Matemáticas, la Ciencia y la Tecnología, que desde hace cuatro años se lleva a cabo en el colegio, asistieron a la conferencia sobre la vida y obra de Darwin llevada a cabo por el Doctor Luís Eugenio Andrade, Vicedecano de la Facultad de Ciencias y profesor titular del Departamento de Biología de la Universidad Nacional de Colombia, sede Bogotá. También tuvieron la oportunidad de apreciar la exposición itinerante titulada Charles Now que recorrió diferentes países de Europa y América, y que organizó el British Council, la cual muestra, a través de pendones muy bien diseñados, aspectos de la vida, experiencias y el legado Charles Darwin. Estas dos actividades fueron un complemento a lo realizado en el salón de clase y también un espacio que permitió generar nuevas inquietudes en los estudiantes.

\section{RESULTADOS Y DISCUSIÓN}

Las actividades propuestas en el desarrollo de este proyecto permitieron que el aula se convirtiera en un espacio donde se posibilitó el habla espontánea, el compartir experiencias, el intercambio de puntos de vista, el construir acuerdos y negociar significados. El proyecto promovió todas las manifestaciones del lenguaje: la oralidad, el dibujo, la lectura y la escritura. Para el docente el proyecto se convirtió en un reto, pues requirió de una fundamentación teórica, de una selección y planeación de las actividades, que permitiera conseguir los objetivos y que además ayudara a mantener el interés de los estudiantes.

Los resultados de esta experiencia permiten sugerir el uso de textos biográficos como una forma distinta de acercar a los estudiantes al llamado conocimiento científico escolar. Sirven como una forma de introducir el tema, pueden usarse como complemento a los contenidos teóricos, se pueden usar para reflexionar y hacer evidente aspectos importantes en el desarrollo de la ciencia, pueden facilitar la identificación y descripción de las dificultades del desarrollo científico en un espacio y contexto geográfico determinado y pueden facilitar el reconocimiento de las incidencias y consecuencias del llamado progreso científico. Se recomiendan entonces los textos biográficos, las entrevistas y las anécdotas; su escogencia depende de los objetivos que el docente desea alcanzar. 
Para el desarrollo de este tipo de experiencia con estudiantes universitarios y de formación disciplinar en ciencias naturales, se sugieren, además de los textos biográficos, las autobiografías y los textos originales. Estos dos últimos, no obstante, también pueden ser usados con estudiantes de educación básica, siempre que el docente planifique su lectura y para una mejor compresión, especialmente si se tiene en cuenta el lenguaje, haga un proceso de adaptación acorde a las necesidades, intereses y objetivos que se tengan. Los aspectos presentados en este trabajo pueden servir de guía para la implementación de esta estrategia en diferentes contextos escolares (Jiménez, 2009).

En esta experiencia se resaltan las bondades del uso de los textos biográficos en el aprendizaje de contenidos conceptuales, metodológicos y actitudinales, pues en ellos se establece una relación estrecha y directa entre el conocimiento científico y su construcción histórica. En concordancia con lo planteado por Velasco, 2008, el uso de biografías en la enseñanza de las ciencias, permite un acercamiento a la historia de la ciencia, lo cual a su vez favorece la comprensión de los contenidos científicos y una visión humana del desarrollo de una disciplina científica.

De otro lado, el desarrollo de este proyecto, además de reconocer los aspectos que posibilitó, que se fueron exponiendo en cada una de las etapas, abrió un espacio para reflexionar sobre tres aspectos fundamentales cuando se usan las biografías como recurso metodológico: el primero, tiene que ver con la necesidad de analizar previamente la coherencia entre los objetivos propuestos y los contenidos históricos que se quieren reconstruir, el segundo, el riesgo que se corre en el uso de las biografías; en su implementación los estudiantes pueden llegar a considerar que el desarrollo científico se debe exclusivamente a personajes especiales y específicos, a los cuales habría que reconocer y admirar. Esta situación genera una tensión entre la singularidad del personaje y todo lo que ello significa, y una perspectiva integral y colectiva del enfoque de la historia de la ciencia. Una manera de aminorar esa posibilidad, es precisamente la de incluir en los espacios de reflexión y discusión, dependiendo el caso, personajes muy cercanos, ya sean con posturas similares o diametralmente opuestas que permitan una visón más amplia del personaje en el contexto del campo del conocimiento en el que él se inscribe. Y el tercero, no menos importante, hace alusión a la planificación de las actividades y su proceso evaluativo; en lo posible deben ser concertadas con los estudiantes; que ellos tengan claro cuáles son los objetivos que se quieren alcanzar.

\section{CONCLUSIONES}

Teniendo en cuenta los objetivos, a continuación se presentan, para cada uno de ellos, los alcances de la experiencia. Como se mencionó al comienzo de este escrito, para los estudiantes el nombre de Darwin no les era desconocido. Pero hay que decir que al final de la experiencia, todos ellos conocían más aspectos sobre su vida personal y familiar, y por supuesto sobre su trabajo y aportes. La descripción que se hizo de los lugares visitados por Darwin en el Beagle les permitió no solo tener una imagen del mismo, sino ir entretejiendo diferentes aspectos que aportaron y fueron la base para lo que hoy se conoce como teoría de la evolución. Esta parte del proyecto favoreció resaltar la importancia que tienen los procesos de observación, descripción, experimentación, comparación y clasificación en un trabajo científico. 
Reconociendo la dificultad que puede tener para estudiantes de grado séptimo comprender claramente los aspectos conceptúales que se derivan de los postulados de Darwin, las actividades que se realizaron, teniendo como punto de partida su biografía, permitieron no sólo introducir el tema, sino acercarse a sus planteamientos de una manera más interesante. En ese sentido vale la pena mencionar que los estudiantes empezaron a reflexionar, entre otras cosas, sobre: la relevancia que tiene la capacidad reproductiva de la especies para dejar descendencia, aquellas que lo hacen tienen mejores procesos de adaptación; la importancia que tienen los recursos alimenticios en ese proceso; la continuidad del proceso evolutivo y su extensión a todos los seres vivos, incluyendo el hombre; y finalmente que los cambios no son perceptibles de una generación a otra, se ha de necesitar mucho tiempo (Pelayo, 2001). De esta manera se resalta la actividad cognitiva del estudiante en la construcción de sus propios conocimientos, pues estos aspectos fueron determinados en los espacios de socialización.

La puesta en marcha de un proyecto como el que se acaba de describir, donde los textos biográficos ${ }^{21}$, las biografías o las autobiografías ${ }^{22}$, pueden servir como hilo conductor, para su implementación es necesario tener presente, además de los objetivos sobre la temática a abordar, los siguientes aspectos:

1. Los objetivos del docente, la temática a desarrollar, los intereses y el grado escolar de los estudiantes. Teniendo en cuenta estos aspectos se determinan: contenidos a abordar de la biografía, su complejidad y el tiempo del proyecto. Es preciso volver a anotar que la profundidad de las reflexiones depende de estos aspectos y de las características de los grupos con quienes se implemente la estrategia.

2. Los saberes de los estudiantes. En ese sentido es importante tener presente dos aspectos: el primero, que los estudiantes traen consigo un cúmulo de conocimientos y experiencias, que son muy valiosas a la hora de iniciar el estudio de un tema; el segundo; no podemos olvidar que la construcción y dominio de un contenido, dentro de cualquier campo de conocimiento, depende de los llamados "conocimientos previos", que luego son reestructurados, reconfigurados o reconstruidos por otros, y que éstos se convierten entonces en el punto de partida de las nuevas experiencias.

3. La selección de las biografías y los contenidos. Estos pueden ser escogidos y demarcados por la relevancia de los aportes del personaje dentro del desarrollo del tema que se está o se quiere abordar, por la cercanía y familiaridad del personaje al contexto en el que el estudiante se encuentra.

21 20. Sobre Darwin hay una gran cantidad de libros. Su selección depende de los objetivos y de la calidad de las fuentes que el autor o autores tome como referentes.

22 19. Al respecto hay que anotar que, aunque para esta experiencia no se leyó el texto completo Autobiografía de Darwin, sino apartes, y básicamente por tiempo, se recomienda su lectura, pues en él se ofrece una visión más amplia de Darwin como persona. Hay que decir que Darwin escribió sus memorias para sus hijos, sin pensar que luego se convertiría en un libro. También se puede encontrar en librodot: http://fierasysabandijas.galeon.com/enlaces/libros/darwauto.pdf. 
4. La selección de los textos. Este aspecto es de vital importancia; deben caracterizarse por tener un manejo adecuado del lenguaje acorde con la edad, brindarles información, pero a su vez permitirles hacer inferencias e hipótesis y generar inquietudes a manera de preguntas. Además, en la selección de los textos, se debe tener en cuenta su extensión, la diagramación, la inclusión de dibujos y cuadros, y la manera como es organizada la información. También en este aspecto es necesario tener presente las características del grupo.

\section{BIBLIOGRAFÍA}

Bachelard, G. (1979). La formación del espíritu científico. Siglo veintiuno Editores Octava Edición.

Briceño, F (2001). La historia de la ciencia como referente en la investigación y didáctica de los contenidos. Aula Ambiente, 1, pp 87-92.

Castro, A. (2008). Las islas Galápagos, Un viaje a través del espacio y el tiempo. Bio-grafia: Escritos sobre la biología y su enseñanza. Vol 1, No. 1.

Castro Moreno, J. (2009). La historia de contingencia Histórica como eje central del Darwinismo. Una discusión en torno a la actualidad de Darwin. Bio-grafia: Escritos sobre la biología y su enseñanza. Vol 2, No 3. Pp.101-123.

Campanario, J.M. (1998). Ventajas e inconvenientes de la Historia de la Ciencia como recurso en la enseñanza de las ciencias. Revista de Enseñanza de la Física, 11, 5-14. http://www.uah.es/otrosweb/jmc

Cerda, H. (2001). El proyecto de aula. El aula como un sistema de investigación y construcción de conocimiento. Bogotá. Cooperativa Editorial Magisterio. pp. 49-97.

Darwin, C. (1859). El origen de las especies. Editorial Planeta-Agostini. Traducción cedida por ediciones Grijalbo. Obras Maestras del Pensamiento Contemporáneo. Edición de 1992. pp. 79-101.

Darwin, C. (1876). Autobiografía. Biblioteca Darwin. Universidad Pública de Navarra. España. Traducción de José Luis Gil. Primera Edición, 2008. pp. 7-124.

Darwin, C. (1939). Diario del viaje de un naturalista alrededor del mundo. Espasa-Calpe. Barcelona, España. Edición 2008. pp. 1-496.

De Santa, A; Martínez F, Mingarro, F, Cárdenas, V; Domínguez, A; Martínez, H y Repetto E. (2005). Albert Einstein entra en la escuela. Utilización de las biografías y de las tecnologías de la información y comunicación en el año internacional de la física. Enseñanza de las ciencias. Número Extra. VII Congreso.

Fernández, M. (2000). Fundamentos históricos. En F.J. Perales y P. Cañal Didáctica de las Ciencias Experimentales, Editorial Marfil: Alcoy. 
Gary, S. (2009). Legado de Darwin. En: Investigación y Ciencia. Enero 2009. No 388. pp 12-17.

Gil, D. (1993). Contribución de la historia y de la filosofía de las ciencias al desarrollo de un modelo de enseñanza/aprendizaje como investigación. Enseñanza de las ciencias. 11, (2), pp. 197-212.

Jiménez, J. (2009). Biografías de científicas. Una aproximación al papel de la mujer en ciencias desde un enfoque socioconstructivista con el uso de las TIC. Rev. Eureka Enseñ. Divul. Cien. 6. pp. 264-277.

Matthews, M. (1994). Historia, filosofía y enseñanza de las ciencias. Enseñanza de las ciencias. 12 (2), pp. 255-277.

Mellado, V y Carracedo, D. (1993). Contribuciones de la filosofía de la ciencia a la didáctica de las ciencias. Enseñanza de las ciencias. 11 (3), pp. 331-339.

Pedrinaci, E. (1996). Por unas fructíferas relaciones entre la historia, la filosofía de la ciencia y la educación científica. Alambique, pp.4-6.

Pelayo, F. (2001). De la creación a la evolución. Darwin. Serie: científicos para la historia. Editorial Nivola ediciones. Pp. 7-72.

Pinilla, R. (2000). Pedagogía de Proyectos y formación de docentes lectores y escritores. En: revista Enunciación. Bogotá, Colombia. pp. 92-94.

Ruiz, C. (2005). Las biografías médicas: ¿estudio de casos para una introducción histórica a la medicina? Asclepio. Vol. LVII. Pp.117-124.

Sánchez, J. (2006). Charles Darwin Alfred y Russel Wallace. La teoría de la evolución de las especies. Clásicos de la ciencia y tecnología. Fundación IBERDROLA. pp. 9-145.

Strathern, P (1999). Darwin y la evolución. Serie. Los científicos y sus descubrimientos. Editorial Siglo Veintiuno Editores S.A. pp.7-89.

Velasco, J. (2008). Historia de la ciencia y enfoque historiográfico en libros de ciencias biológicas de educación básica y educación diversificada profesional en Venezuela. Revista de investigación No 64. pp. 63-84.

Villar, C (2004). La formación de docentes investigadores. Colección Investigación y Enseñanza. Serie Fundamentos No 21. 